Elsevier

BPC 01180

\title{
Time-dependent ligand current into a single cell performing chemoreception
}

\author{
Bernard J. Geurts and Frederik W. Wiegel \\ Center for Theoretical Physics, University of Twente, P.O. Box 217, Enschede 7500 AE, The Netherlands \\ Received 27 April 1987 \\ Accepted 5 June 1987 \\ Chemoreception; Boundary condition approach
}

\begin{abstract}
We determine the ligand current into a single spherical cell which carries a large number of receptors on its surface. Initially, this cell is placed into a medium which contains ligands at uniform concentration. The time-dependent ligand distribution is calculated, from which the time-dependent ligand current into the cell is derived. If the ligand concentration is kept constant at distances comparable to the radius of the cell the stationary state sets in at times comparable to the time $T_{1}$ necessary for ligands to travel a distance of the order of the radius of the cell. If the ligand concentration is kept constant at infinity the stationary state sets in at a time which is about $1000 T_{1}$ for typical values of the parameters.
\end{abstract}

\section{Introduction}

The problem of chemoreception of ligands by living cells has received considerable theoretical attention in recent years [1-3]. It is closely related to a calculation of the rate constant in chemical reactions $[4,5]$. In this paper we consider the problem in which a cell with a large number of receptors on its surface is placed at $t=0$ in a medium which contains ligands at uniform concentration. We shall solve this time-dependent problem using the boundary condition approach of Delisi and Wiegel [7] (cf. also ref. 1).

The cell is modeled as a sphere of radius $R$, on which $N \gg 1$ ideal receptors for ligands are distributed. These receptors are assumed to be characterized by a single linear dimension s. In the exterior medium the ligands follow diffusive paths, and their translational motion is characterized by a diffusion coefficient $D$. In general, not every encounter of a ligand with the cell will lead to

Correspondence address: B.J. Geurts, Center for Theoretical Physics, University of Twente, P.O. Box 217, Enschede 7500 $A E$, The Netherlands binding of the ligand. This can be modeled $[1,7]$ by taking the ligand concentration on the cell surface to be proportional to the gradient of this concentration on the cell surface. The proportionality constant $\alpha$ is a function of $R, N$ and $s$.

Many actions of living cells are regulated by the flow of certain ligands into the cell. This information current depends on the concentration gradient at the cell's surface which approaches a stationary value which depends on the parameters. It is shown that, at typical parameter values, the stationary state sets in on a time scale $T_{s}$ which is comparable to the time $T_{1}$ necessary for a ligand to travel the distance of a cell, provided the ligand concentration is kept constant at a distance comparable to the radius of the cell. If the ligand concentration is kept constant at large distances from the cell the relaxation time $T_{\mathrm{s}}$ is about 1000-times as large as $T_{1}$.

In section 2 we formulate the problem in terms of the diffusion equation. Section 3 is devoted to the case where the ligand concentration is kept fixed at some finite distance $l$. The case in which $l$ goes to infinity is treated in section 4 , and some concluding remarks are collected in section 5 . 


\section{Formulation of the problem}

Consider a spherical cell of radius $R$, placed in a medium in which at $t=0$ ligands are distributed uniformly. The cell carries $N$ receptors in its outer membrane which are specific for the ligands; the size of the receptor can be characterized by a single length $s$ [1]. The ligands in the medium follow diffusive paths and their translational motion can be characterized by a diffusion coefficient $D$.

The time evolution of the number density of ligands outside the cell $c$ is governed by the diffusion equation which, in view of the spherical symmetry of the problem, can be written as

$\frac{1}{D} \partial_{t} c(r, t)=\partial_{r r} c(r, t)+\frac{2}{r} \partial_{r} c(r, t)$

where $r$ measures the distance from the center of the cell and $t$ is the time. Initially the ligand distribution is homogeneous, so everywhere in the medium outside the cell

$c(r, 0)=c_{0}$

where $c_{0}$ is some constant ligand concentration. At some distance $l>R$ from the center of the cell the ligand concentration is assumed to be kept constant and equal to the initial ligand density

$c(l, t)=c_{0}(t \geq 0)$

The boundary condition on the cell surface can be taken as $[1,7]$

$c-\alpha \partial_{r} c=0$ for $r=R$ and $t \geq 0$

where [1]

$\alpha=\frac{\pi R^{2}}{N s}$

i.e., the ligand density at the surface is proportional to its gradient. This boundary condition expresses the fact that not every encounter of a ligand with the cell surface leads to the capture of the ligand by the cell. If $\alpha=0$ the entire cell membrane acts as an ideal receptor; in the limit $\alpha \rightarrow \infty$ the cell catches no ligands at all.

It is straightforward to find the stationary-state solution to the problem posed by eqs. 1-4

$c(r)=c_{0} \frac{l(R+\alpha)}{l(R+\alpha)-R^{2}}\left[1-\frac{R}{R+\alpha} \frac{R}{r}\right]$

Guided by this we use the following representation for the time-dependent solution

$$
\begin{aligned}
c(r, t)= & c_{0} \frac{l(R+\alpha)}{l(R+\alpha)-R^{2}} \\
& \times\left[1-\frac{R}{R+\alpha} \frac{R}{r}(1-f(r, t))\right]
\end{aligned}
$$

Upon substitution of eq. 6 into eq. 1 one finds that $f(r, t)$ must be a solution of

$\frac{1}{D} \partial_{t} f(r, t)=\partial_{r r} f(r, t)$

The initial and boundary conditions for $f(r, t)$ are

$f(r, 0)=\frac{l-r}{l}(R<r<l)$

$f(l, t)=0(t \geq 0)$

$f-\frac{\alpha R}{R+\alpha} \partial_{r} f=0$ for $r=R$ and $t \geq 0$

Note that with the representation, eq. 6 , the ligand distribution $c(r, t)$ is obtained from the solution of the simpler one-dimensional problem posed by eqs. 7 and 8 for $f(r, t)$ [5].

In section 3 we shall give the solution to the above problem and calculate the ligand current $J(t)$ into the cell from

$J(t)=D \int_{s} \int_{D c} \mathrm{~d} S=4 \pi R^{2} D \partial_{r} c$ for $r=R$

where $S$ is the surface of the cell. The physically most realistic case in which $l \rightarrow \infty$ will be treated separately in section 4 .

\section{Ligand distribution and current for the general case}

The problem as posed by eqs. 7 and 8 can be solved analytically by separating variables. The 
eigenfunctions of eq. 7 are

$$
\begin{aligned}
\varphi_{n}(r, t)= & {\left[A_{n} \sin \left(\lambda_{n}(r-R)\right)\right.} \\
& \left.+B_{n} \cos \left(\lambda_{n}(r-R)\right)\right] e^{-\lambda_{n}^{2} D t}
\end{aligned}
$$

In view of the conditions, eqs. $8 \mathrm{~b}$ and $8 \mathrm{c}$, one obtains the following consistency relation for the coefficients $A_{n}, B_{n}$ and the eigenvalues $\lambda_{n}$

$B_{n}=\frac{\lambda_{n}}{\beta} A_{n}$

$\tan \left(\lambda_{n}(l-R)\right)=-\frac{\lambda_{n}}{\beta}$

The parameter $\beta$ is defined as

$\beta=\frac{R+\alpha}{\alpha R}$

Since the problem is linear and homogeneous, one thus obtains for the general solution

$$
\begin{aligned}
f(r, t)= & \sum_{n=1}^{\infty} \frac{A_{n}}{\beta}\left[\lambda_{n} \cos \left(\lambda_{n}(r-R)\right)\right. \\
& \left.+\beta \sin \left(\lambda_{n}(r-R)\right)\right] e^{-\lambda_{n}^{2} D t}
\end{aligned}
$$

where the eigenvalues $\lambda_{n}>0$. They follow from the transcendental equation (eq. 12) and cannot be determined analytically. It is, however, possible to express the coefficients $A_{n}$ in terms of the eigenvalues $\lambda_{n}$, and hence $f(r, t)$ can be determined with arbitrary accuracy from a numerical determination of the eigenvalues using eq. 12.

The coefficients $A_{n}$ follow from matching eq. 14 to the initial condition (eq. 8a)

$$
\begin{gathered}
\sum_{n=1}^{\infty} \frac{A_{n}}{\beta}\left[\lambda_{n} \cos \left(\lambda_{n}(r-R)\right)+\beta \sin \left(\lambda_{n}(r-R)\right)\right] \\
\quad=\frac{l-r}{l} \text { for } R<r<l
\end{gathered}
$$

The following orthogonality relations hold

$$
\begin{aligned}
& \int_{R}^{l} g_{m}(r) g_{n}(r) \mathrm{d} r \\
& \quad=0, m \neq n \\
& \quad=\frac{2 \beta}{\left(\lambda_{n}^{2}+\beta^{2}\right)(l-R)+\beta}, m=n
\end{aligned}
$$

where

$$
g_{n}(r)=\lambda_{n} \cos \left(\lambda_{n}(r-R)\right)+\beta \sin \left(\lambda_{n}(r-R)\right)
$$

and the $\lambda_{n}$ satisfy eq. 12 . Hence

$$
\frac{A_{n}}{\beta}=\frac{2}{\left(\lambda_{n}^{2}+\beta^{2}\right)(l-R)+\beta} \frac{1}{\lambda_{n}}\left[\frac{\beta(l-R)+1}{l}\right]
$$

The solution for $f(r, t)$ is obtained from inserting eq. 18 into eq. 14. In fig. 1 the ligand distribution $c(r, t)$ is shown, at $\alpha=1 \times 10^{-6} \mathrm{~m}, R=5 \times 10^{-6}$ $\mathrm{m}$ and $l=25 \times 10^{-6} \mathrm{~m}$. A typical value for $D$ is $10^{-10} \mathrm{~m}^{2} \mathrm{~s}^{-1}$, so it takes $T_{1} \approx 0.25 \mathrm{~s}$ for ligands to travel the distance of a cell. These parameter values were taken from ref. 1. At these parameter values the stationary state sets in at a time scale $T_{s}$ comparable to the time necessary for ligands to travel the distance of a cell $\left(T_{\mathrm{s}} \approx 6 T_{1}\right)$. We will return to this point shortly.

One can now determine the ligand current into the cell from eqs. 6 and 9: this gives

$$
\begin{aligned}
I(t)= & 4 \pi R D c_{0} \frac{l R}{l(R+\alpha)-R^{2}} \\
& \times\left[1+\frac{R}{\alpha} \sum_{n=1}^{\infty} \lambda_{n} \frac{A_{n}}{\beta} e^{-\lambda_{n}^{2} D_{t}}\right]
\end{aligned}
$$

which implies for the stationary state

$J_{\alpha}(l)=4 \pi R D c_{0} \frac{l R}{l(R+\alpha)-R^{2}}$

In the limit $l \rightarrow \infty$ this result combined with eq. $4 a$ for the parameter $\alpha$ yields a stationary ligand current identical with the result of ref. 3 .

In fig. $2 J(t) / J_{0}(l)$ is shown for some choices of $\alpha$ and $l$, at $R=5 \times 10^{-6} \mathrm{~m}$. The quantity $J_{0}(l)$ is the stationary ligand current for the case of a perfectly absorbing sphere $(\alpha=0)$ with fixed concentration at $r=l$. Note that as $\alpha$ is increased, i.e., the average number of encounters of a ligand with the cell before reception increases, $J(t) / J_{0}(l)$ decreases drastically. Also, if $l$ is increased at fixed $\alpha$ and $R$ values, $J(t) / J_{0}(l)$ increases. From this figure one also recognizes that as $l$ increases it takes longer and longer hefore the stationary state 


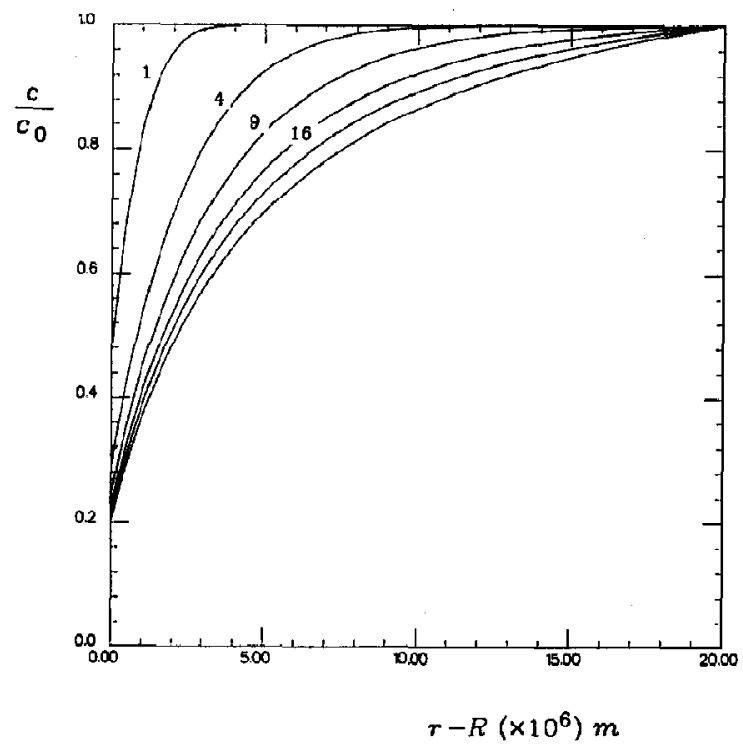

Fig. 1. Ligand distribution at $l=25 \times 10^{-6} \mathrm{~m}, R=5 \times 10^{-6}$ $\mathrm{m}$, and $a=1 \times 10^{-6} \mathrm{~m}$. The index on the lines denotes the corresponding value for $D t$ in units of $10^{-12} \mathrm{~m}^{2}$.

sets in. In order to make this statement quantitative, we have shown in fig. 3 a plot of $\log _{10}\left(T_{s} / T_{1}\right)$ as a function of $\alpha$ for various values of $l$. Here, $T_{s}$

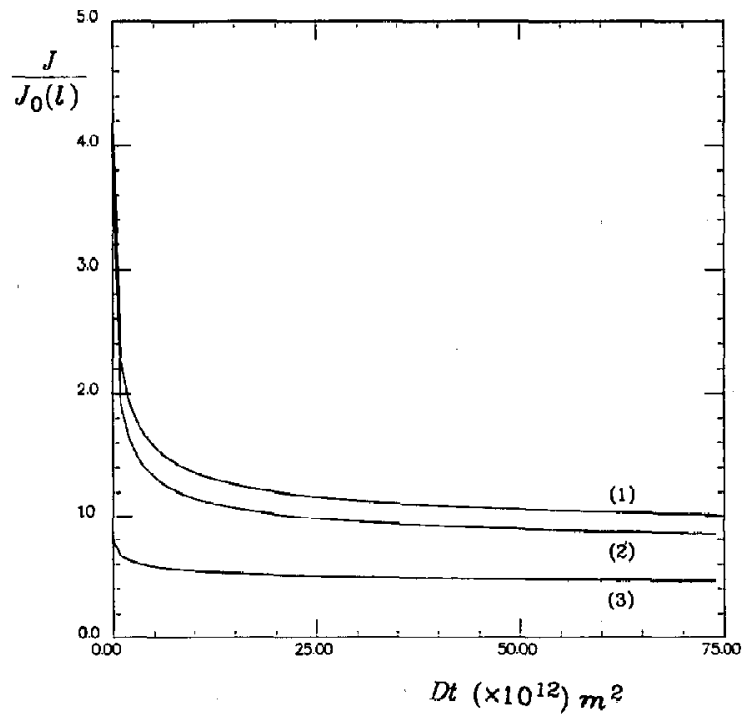

Fig. 2. Ligand current ratio $J(t) / J_{0}(l)$, at $R=5 \times 10^{-6} \mathrm{~m}$ and (1) $(l, \alpha)=\left(100 \times 10^{-6} \mathrm{~m}, 1 \times 10^{-6} \mathrm{~m}\right),(2)(l, \alpha)=\left(25 \times 10^{-6}\right.$ $\left.\mathrm{m}, 1 \times 10^{-6} \mathrm{~m}\right),(3)(1, \alpha)=\left(25 \times 10^{-6} \mathrm{~m}, 5 \times 10^{-6} \mathrm{~m}\right)$.

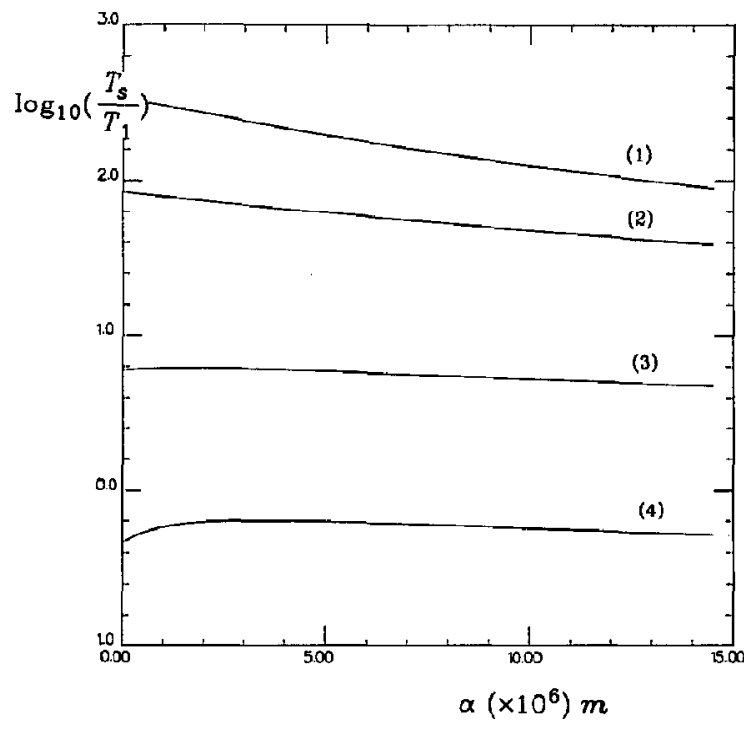

Fig. 3. The logarithm of the ratio of the stationary-state time $T_{\mathrm{s}}$ and the time necessary for ligands to travel a distance of the order of the radius of the cell, $T_{1}$, as a function of $\alpha$ at various $l$ values; (1) $l=250 \times 10^{-6} \mathrm{~m}$, (2) $l=100 \times 10^{-6} \mathrm{~m}$, (3) $l=25$

$$
\times 10^{-6} \mathrm{~m} \text { and }(4) l=10 \times 10^{-6} \cdot \mathrm{m} \text {. }
$$

is the time necessary for the stationary state to set in; as a measure for this we used

$$
\frac{J\left(T_{\mathrm{s}}\right)-J_{\alpha}(l)}{J_{\alpha}(l)}=x
$$

where $x=O\left(10^{-2}\right)$. As $l$ is of the order of the radius of the cell $T_{\mathrm{s}}$ is of the order of $T_{1}$. If $l / R$ is increased to one or two orders of magnitude $T_{\mathrm{s}}$ is of the order of 100-1000-times $T_{1}$ at typical parameter values. An interesting aspect is that as $l / R=O(1)$ the ratio $T_{\mathrm{s}} / T_{1}$ decreases slightly as $\alpha$ decreases to zero.

\section{Ligand distribution and ligand current for the case $l \rightarrow \infty$}

The problem as posed by eqs. 7 and 8 can be treated fully analytically in the limit $l \rightarrow \infty$. A closed expression for $f(r, t)$ can be found using the heat kernel method [6]. One straightforwardly 
shows that, with $y=r-R$ and $T=D t$

$$
\begin{aligned}
& f(y, T) \\
& \quad=\int_{0}^{\infty}[k(y-\xi, T) \\
& \left.\quad+\left(1-2 \beta e^{-\beta \xi} \int_{0}^{\xi} e^{\xi^{\prime}} \mathrm{d} \xi^{\prime}\right) k(y+\xi, T)\right] \mathrm{d} \xi
\end{aligned}
$$

where $k\left(z_{1}, z_{2}\right)$ is the heat kernel

$$
k\left(z_{1}, z_{2}\right)=\frac{1}{2\left(\pi z_{2}\right)^{1 / 2}} \exp \left[-\frac{z_{1}^{2}}{4 z_{2}}\right]
$$

Evaluation of the integral, eq. 22, gives

$$
\begin{aligned}
f(r, t)= & \operatorname{erf}\left[\frac{r-R}{2(D t)^{1 / 2}}\right]+e^{\beta(r-R)+\beta^{2} D t} \\
& \times \operatorname{erfc}\left[\frac{r-R}{2(D t)^{1 / 2}}+\beta(D t)^{1 / 2}\right]
\end{aligned}
$$

where $\operatorname{erf}(y)$ is the error function and $\operatorname{erfc}(y)$ the complementary error function (cf. eqs 7.1.1 and 7.1.2 in ref. 8). The ligand distribution $c(r, t)$ is similar to that for finite $l$ values and is not repeated here.

Using eq. 9, one finds for the ligand $J(t)$

$$
\begin{aligned}
J(t)= & 4 \pi R D c_{0} \frac{R}{R+\alpha} \\
& \times\left[1+\frac{R}{\alpha} e^{\beta^{2} D t} \operatorname{erfc}\left(\beta(D t)^{1 / 2}\right)\right]
\end{aligned}
$$

with the stationary value

$J_{\alpha}=4 \pi R D c_{0} \frac{R}{R+\alpha}$

The ratio $J(t) / J_{0}$ depends on $D t$ in a manner quite similar to that shown in fig. 2 . The only essential difference is that the time $T_{s}$ is much larger than in the case where $l=O(R)$. As $l \rightarrow \infty$ the time $T_{\mathrm{s}}$ is given implicitly by

$$
\frac{R}{\alpha} \exp \left(\beta^{2} D T_{s}\right) \operatorname{erfc}\left(\beta\left(D T_{s}\right)^{1 / 2}\right)-x=0
$$

where $x=O\left(10^{-2}\right)$. In the case $\beta\left(D T_{s}\right)^{1 / 2} \gg 1$ one can use the asymptotic behavior (cf. eq. 7.1.23 of ref. 8)

$$
\begin{gathered}
\exp \left[\beta^{2} D T_{\mathrm{s}}\right] \operatorname{erfc}\left(\beta\left(D T_{\mathrm{s}}\right)^{1 / 2}\right) \\
\approx\left(\pi^{1 / 2} \beta\left(D T_{\mathrm{s}}\right)^{1 / 2}\right)^{-1}
\end{gathered}
$$

and find

$$
\frac{\pi x^{2} T_{s}}{T_{1}} \approx \frac{R^{2}}{(R+\alpha)^{2}}
$$

The correspondence between the asymptotic result (eq. 29) and the exact numerically determined result based on eq. 27 is almost perfect for all realistic $\alpha$ values, since eq. 28 is already very accurate if $\beta\left(D T_{s}\right)^{1 / 2}>5$. In addition, $\pi x^{2} T_{s} / T_{1}$ depends very little on $x$ for $x<0.05$. As $\alpha \rightarrow 0$, $T_{s}=O\left(10^{3}\right) T_{1}$, viz., of the order of $4 \mathrm{~min}$. $T_{s}$ decreases continuously as $\alpha$ is increased.

Using eq. $4 \mathrm{a}$, the ratio of $J(t)$ to the stationary ligand current for a perfectly absorbing sphere $J_{0}$ can be written in the form

$$
\begin{aligned}
\frac{J(t)}{J_{0}} & =\frac{1}{1+\mu}\left[1+\frac{1}{\mu} e^{\beta^{2} D t} \operatorname{erfc}\left(\beta(D t)^{1 / 2}\right)\right] \\
\mu & =\frac{\pi R}{N s}
\end{aligned}
$$

This implies for the $t \rightarrow 0$ and $t \rightarrow \infty$ limits that

$$
\begin{aligned}
& \lim _{t \rightarrow 0} \frac{J(t)}{J_{0}}=\frac{1}{\mu} \\
& \lim _{t \rightarrow \infty} \frac{J(t)}{J_{0}}=\frac{1}{1+\mu}
\end{aligned}
$$

At typical parameter values taken from ref. 1, $N \approx 10^{4}, s \approx 5 \times 10^{-9} \mathrm{~m}$ one has $\mu \approx 0.3$. Hence, the stationary-state ligand current is about 4-times as low as its initial value. It is remarkable that the boundary condition approach $[1,7]$ gives correct results in the $t \rightarrow 0$ limit, since it was derived from asymptotic matching considerations.

\section{Concluding remarks}

We have obtained the time-dependent solution of the ligand distribution and ligand current into a spherical cell with $N$ ideal receptors in its mem- 
brane. The time scale for a ligand to travel the distance of a cell $T_{1}$ is about $0.25 \mathrm{~s}$. It was shown that the stationary state sets in at a time scale $T_{\mathrm{s}}$ of the order of $T_{1}$ if the ligand concentration is kept constant at distances $l$ which are comparable to the radius of the sphere (i.e., $l$ has the same order of magnitude as $R$ ). If the ligand concentration is kept constant at much larger distances $T_{s} / T_{1}$ is of the order $10^{3}$.

\section{Acknowledgement}

One of the authors (F.W.W.) would like to acknowledge useful discussions about chem- oreception with Dr. John C. Owicki of the University of California at Berkeley.

\section{References}

I F.W. Wiegel, Phys. Rep. 95 (1983) 283.

2 O.G. Berg, Biophys. J. 47 (1985) 1.

3 H.C. Berg and E.M. Purcell, Biophys. J. 20 (1977) 193.

4 G.H. Weiss, J. Stat. Phys. 42 (1986) 3.

5 H.S. Carslaw and J.C. Jaeger, Conduction of heat in solids (Clarendon Press, Oxford, 1959).

6 E.T. Copson, Partial differential equations (Cambridge University Press, Cambridge, 1975).

7 C. DeLisi and F.W. Wiegel, Proc. Natl. Acad. Sci. U.S.A. 78 (1981) 5569.

$8 \mathrm{M}$. Abramowitz and I.A. Stegun, Handbook of mathematical functions (Dover, New York, 1964). 